\title{
Plasmablastic myeloma versus plasmablastic lymphoma: different yet related diseases
}

\begin{abstract}
We describe a case of ileal plasmablastic myeloma in a 77-year-old otherwise immunocompetent male patient. The patient presented with weight loss, abdominal pain, and loose stools. Imaging studies demonstrated wall thickening in the distal ileal loop. He underwent ileocolectomy and pathology was consistent with plasmablastic neoplasm. Subsequently, the pathologic evaluation was felt to be consistent with a small bowel plasmablastic myeloma that was EBV positive, C-MYC negative, CD56 and CD138 positive, Ki-67 score greater than $90 \%$, and lambda light chain mRNA restricted.

Plasmablastic myeloma is a rare and aggressive neoplasm with a poor prognosis. Similarly, plasmablastic lymphoma is also a rare, aggressive, and typically fatal disease that is often associated with HIV. Both PBL and PBM have several overlapping cytomorphologic and immunophenotypic features, making a precise diagnosis difficult to provide to patients. Immunophenotypically, CD38 and CD138 positivity is unreliable to differentiate between PBL and PBM.

Plasmablastic myeloma tends to have worse outcomes than other plasma cell dyscrasias. Moreover, there is very limited data on plasmablastic myeloma of the small bowel and treatment recommendations are based solely upon antidotal experience. Molecularly, there is information that plasmablastic myelomas and lymphomas are related neoplasms, which indicates that there may be treatment overlap between these two neoplasms.
\end{abstract}

Keywords: plasmablastic myeloma, plasmablastic lymphoma, MYC, PRDM1, MLL, FISH
Volume 6 Issue I - 2018

\author{
Ann Morris,' Gregory Monohan ${ }^{2}$ \\ 'College of Medicine Phoenix, University of Arizona, USA \\ ${ }^{2}$ Department of Medicine, University of Kentucky, USA
}

\begin{abstract}
Correspondence: Gregory Monohan, University of Kentucky, Department of Medicine, Division of Hematology/BMT, CC4I4, 800 Rose Street, Lexington, KY 40536, USA, Fax 859.257.7715, Tel 859.323.5877, Email monohan@uky.edu
\end{abstract}

Received: August 08, 2017 | Published: February 02, 2018

\section{Introduction}

Plasmablastic myeloma (PBM) is a rare, monoclonal, immunoproliferative plasma cell neoplasm that shares many characteristics with plasmablastic lymphoma (PBL). ${ }^{1}$ Currently, there are no set standards for differentiating between PBL and PBM. Morphologically, PBM is characterized by the presence of large plasma cells with large hyperchromatic nuclei, one or more prominent centrally-located nucleoli, a rim of basophilic cytoplasm, high nuclear/ cytoplasmic ratio, and an increased number of mitoses. ${ }^{2}$ Plasmablastic myeloma is more likely to express CD38 and CD138 with light chain restriction. ${ }^{3}$ Yet, in a small study of 16 patients ( 9 cases of PBL and 7 cases of PBM), all cases had immunochemistries positive for MUM1/IRF4, CD138, and CD38, and negative for CD20. ${ }^{1}$ Indicating that simply using CD38 and CD138 positivity to differentiate between PBL and PBM is unreliable (Table 1). Moreover, another study concluded that a patient with PBM was positive for CD38 and MUM1, but negative for $\mathrm{CD} 138 .{ }^{2}$ Thus, there are conflicting reports for the ability of immunohistochemical studies to differentiate between PBL and PBM.

Table I Indicating that using CD38 and CDI38 positivity to differentiate between PBL and PBM

\begin{tabular}{lll}
\hline & Plasmablastic lymphoma & Plasmablastic myeloma \\
\hline Morphology & $\begin{array}{l}\text { Large B lymphocytes with loss of general B-cell } \\
\text { antigens and expression of plasma cell phenotype. } \\
\text { Proliferation of plasmablasts and/or immunoblasts, } \\
\text { with rare plasmacytic cells, EBV infection often } \\
\text { associated. }\end{array}$ & $\begin{array}{l}\text { Presence of large plasma cells, often containing } \\
\text { prominent nucleoli, hyperchromatic nuclei and increased } \\
\text { nuclear to cytoplasmic ratio with increased mitotic } \\
\text { activity. }\end{array}$ \\
Immunohistochemistry & CD38, CDI38, XBPI, PRDMI/BlimpI,VEGF,Vimentin & CD38, CDI38, light chain restriction. \\
Genetic mutation reported* & C-MYC, PRDMI, EBER & BRAF, C-MYC, P53, MLL, ETV6, t(I4:I8) \\
\hline
\end{tabular}

Genetic analyses for PBM are lacking in the literature. Although there is a case report of plasma cell myeloma with plasmablastic differentiation that showed genetic mutations in BRAF via immunohistochemical studies, multiple copies of MLL and ETV6 genes via FISH, and mutations in C-MYC, p53, and Ki67. ${ }^{4}$ In one study of 14 patients with B-cell neoplasms, 1 was found to have PBM with expression of BCL-2, a proliferation index of $80 \%$ (Ki-
67), and translocation $\mathrm{t}(14 ; 18) .{ }^{5}$ In this small study, the PBM tumor cells were positive for CD33, CD38, CD138, and lambda light chain, but negative for CD13, CD20, CD34, CD56, CD117, and TdT. ${ }^{5}$ Yet, studies of specifically plasmablastic myeloma genetics will be required to elucidate the underlying similarities and differences from PBL. 
Plasmablastic lymphoma (PBL) is a rare, aggressive subtype of non-Hodgkin's lymphoma (NHL) with a relatively recent discovery in $1997 .{ }^{6}$ Initially, it was thought be related to HIV infection and limited to neoplasms of the oral cavity and jaw. Although more recent studies suggest that PBL can present in the gastrointestinal tract as well and that iatrogenic immunosuppression or immunosenescence can predispose patients to PBL. ${ }^{7}$ Although EBV infection is found in a majority of cases, it is not required for diagnosis of PBL. Furthermore, recent evidence suggests that EBV and HIV may not influence gene expression in the case of PBL. ${ }^{6}$

PBL is a high-grade large B-cell neoplasm with plasma cell phenotype with loss of B-cell antigens, down-regulation of PAX5 \& CD20, and over expression of XBP1 \& PRDM1/Blimp $1 .{ }^{6}$ Genetic mutations in MYC occur in a majority of PBL cases (approximately $60 \%$ ) with the most common alteration being a translocation between MYC and IgH. ${ }^{6} \mathrm{MYC}$ over expression is not limited to translocations, but does play an important role in PBL. ${ }^{6}$ Another key gene in PBL is PRDM1, which expresses Blimp1 protein, acts as tumor suppressor, and is a regulator of plasma cell differentiation. ${ }^{6}$ It is over expressed in most PBLs. Both MYC and PRDM1 play a role in inducing terminal differentiation and growth arrest in B cells. ${ }^{6}$ In one study of 36 PBL cases, PRDM1 missense mutations are common in PBL and are associated with terminal B-cell differentiation. ${ }^{6}$ This same study demonstrated that in 24 samples studied by RT-PCR there were undetectable levels of PRDM1-beta transcripts. Rather they express full-length PRDM1-alpha transcripts, unlike myeloma cell lines. ${ }^{6}$ Thus, PRDM1 and MYC play an important role in the genetic aberrations leading to PBL.

\section{Case presentation}

The patient is a 77-year-old man with history of hypertension, benign prostatic hypertrophy, and osteoarthritis, who presented to clinic with plasmablastic myeloma of the ileum. Initially, the patient reported a 33-pound weight loss with associated abdominal pain and frequent loose bowel movements in the summer of 2016. Occasionally, his bowel movements contained blood. At that time, he was evaluated for possible Crohn's disease, but colonoscopy was negative. His upper endoscopy demonstrated a gastric ulcer that was cauterized. The abdominal x-rays demonstrated a density projecting over the distal ileum and a CT scan of his abdomen and pelvis demonstrated what appeared to be wall thickening in the distal ileal loop. Subsequently, the patient underwent ileocolectomy and pathology was consistent with plasmablastic neoplasm with negative lymph node involvement. Pathology demonstrated Ki-67 score greater than 90\%, CD56 and CD138 positivity on immunohistochemistry, and Lambda light chain restriction. The diagnosis of plasmablastic myeloma was favored over plasmablastic lymphoma based on these findings.

The patient underwent further evaluation and was found to have a beta-2 microglobulin level of 2.7, serum free kappa light chain of $29.8 \mathrm{mg} / \mathrm{L}$, and serum free lambda light chain of $23.5 \mathrm{mg} / \mathrm{L}$ with a ratio of 1.27 . The serum protein electrophoresis was negative. The 24-hour urine showed $79.2 \mathrm{mg}$ total protein/day and was negative for immunofixation electrophoresis and Bence-Jones proteins. Together, these findings favor a diagnosis of PBM over multiple myeloma. Additional diagnostic findings are reported under the results section.

Following the patient's initial diagnosis, he was referred to an academic medical center. At that time, the patient's appetite and weight had stabilized. He continued to report intermittent diarrhea, but it had decreased since the ileocolectomy and no longer contained blood. The patient continued to report chronic fatigue, back pain, and arthralgias without abdominal pain. He denied any fevers, night sweats, adenopathy, or persistent infections.

The patient's final diagnosis was plasmablastic myeloma of the small bowel that is EBV positive, MYC negative, CD56 and CD138 positive, Ki-67 score greater than $90 \%$, and lambda light chain mRNA restricted. The patient understood the significance of his diagnosis and the limited research available in the medical literature. It was explained that the patient was diagnosed with a rare type of aggressive blood cancer that has features between multiple myeloma and aggressive lymphoma. This diagnosis tends to have worse outcomes than other plasma cell dyscrasias. Moreover, there is very limited data on plasmablastic myeloma of the small bowel and treatment recommendations are based solely upon antidotal experience. In this case, treatment decisions were based upon the extent of disease and a PET scan was recommended. If the PET scan demonstrated systemic disease, dose adjusted-EPOCH (etoposide, doxorubicin, cyclophosphamide, vincristine, prednisone) with Bortezomib (Velcade) would be recommended, ${ }^{8}$ which is a treatment modality often utilized in plasmablastic lymphoma. If the PET scan were negative, a Bortezomib -based Myeloma treatment plan was recommended. Finally, from a molecular perspective, there is information that plasmablastic myelomas and lymphomas are related diseases neoplasms, ${ }^{1}$ which may indicate that there may be treatment overlap between these two neoplasms. After evaluation was complete, our patient had no systemic evidence of disease and began Lenolidomide, Bortezomib and Dexamethasone chemotherapy with plans to transition to maintenance Lenolidomide after several cycles of treatment. At time of article submission, the patient was in remission and tolerating Lenolidomide maintenance therapy nine months after diagnosis.

\section{Results}

\section{Histopathological findings}

Histologically, sections of small bowel tumor showed a diffuse, transmural proliferation of plasmablastic cells. Frequent mitoses and apoptotic bodies were noted. Pathology reported that the small bowel tumor resection was positive for plasmablastic neoplasm. The peripheral blood smear showed normocytic anemia with rare elliptocytes and schistocytes without evidence of rouleaux formation. There was no evidence of circulating blasts. The bone marrow cellularity was normocellular for age (30\% cellularity) without evidence for metastatic carcinoma, sarcoma, lymphoma, or granulomas.

\section{Immunohistochemical findings}

The tumor cells had a nearly $100 \% \mathrm{Ki}-67$ index. The neoplasm was negative for CD19, PAX-5, CD20, CD34, CD30, HHV-8, cytokeratin AE1/AE3, Cam 5.2, synaptophysin, chromogranin, S100, HMB-45, MART-1, SOX-10, PSA, PSAP, and MYC. The tumor cells were positive for CD56, CD138, and Lambda mRNA restricted. Flow cytometry of bone marrow showed phenotypically normal, left-shifted myeloid cells without flow cytometric evidence of monoclonality, acute leukemia, plasma cell dyscrasia, or lymphoproliferative disorder.

Analysis of the bone marrow aspirate showed $14 \%$ of the CD45+leukocytes are lymphocytes, $2 \%$ were monocytes, and $84 \%$ were myeloid cells. Eighty percent of the lymphocytes were T-cells, $10 \%$ were NK cells, and 4\% were B cells. The T-cells appeared phenotypically normal with a CD4: CD8 ratio of 2.9. The B-cells 
were polytypic and had a kappa: lamba ratio of 1.0. Rare plasma cells expressed bright $\mathrm{CD} 38$, dim to negative $\mathrm{CD} 45$, and polytypic cytoplasmic light chains, without CD56, representing approximately $0.7 \%$ of the leukocytes. The myeloid cells demonstrated phenotypically normal expression patterns for the markers evaluation, including CD10, CD13, CD16, CD33, and CD64, with a left shift (increased percentage that lacked CD11b), and included 2\% CD34+ myeloblasts.

\section{In situ hybridization}

The tumor cells were positive for Epstein-Barr virus (EBV) and co-expressed EBV and CD56.

\section{FISH analysis}

Fluorescence in situ hybridization (FISH) was performed on 200 interphase cells and scored for each of the following probe combinations: CKS1B, TP53 (Cytocell), ASS1, CCND1/IGH XT, IGH, and PML on CD138+ sorted cells. FISH analysis was also performed with the BCL6, MYC, IGH, and BCL2 probes on 200 interphase cells. FISH analysis showed normal results without evidence of gain of CKS1B at 1q, an IGH rearrangement, TP53 deletion, or gain of chromosomes 9,11 , or 15 . The second analysis showed normal results with no evidence of $t(14 ; 18)$ (q32;q21) (IGH/BCL2 translocation) or other rearrangements involving BCL6 or MYC. Myeloma and lymphoma FISH panels were reportedly negative.

\section{Imaging studies}

The skeletal bone survey was negative for lytic bone disease. The $\mathrm{CT}$ scan of abdomen/pelvis was positive for distal ileac loop wall thickening.

\section{Conclusion}

Both PBM and PBL are rare diseases that are difficult to diagnose and carry with them a poor prognosis. The PBM case presented here was a primary small bowel lymphoma of a HIV-negative, EBV positive, c-MYC negative, CD56 and CD138 positive, high proliferation index, and lambda light chain mRNA restricted patient with morphologic and phenotypic features of PBM. There are several difficulties in reliably differentiating between these two neoplasms, including their morphologic, cytologic, and immunophenotypic similarities. Morphologic differences include PBLs with a proliferation of plasmablasts and/or immunoblasts, with rare plasmacytic cells, while PBMs often have plasmacytic cells. ${ }^{1}$ Yet, these differences are subtle and the neoplasms are otherwise very similar. Thus, without a means of further differentiating these two neoplasms, relying solely on morphological features may not allow clinicians to consistently separate these two diagnoses.

In a small study of 9 plasmablastic lymphoma and 7 plasmablastic myeloma patients, it was shown that these patients had comparable genetic alterations with some subtle differences. ${ }^{1}$ Both had a high proliferation index and similar tumor gene expression profiles, with loss of p16 and p27, and positive expression of p53. In situ hybridization assays showed that EBER was positive in 5 of the PBL cases and negative in all of the PBM cases. ${ }^{1}$ EBER status has been shown in other studies to be able to differentiate between PBL and PBM, but recent studies are lacking. ${ }^{910}$ Finally, EBV status has been shown to be more highly associated with PBL than PBM, yet this was not seen in our patient. ${ }^{1}$ Although it has been indicated that EBV status can help differentiate between PBL and PBM, ${ }^{5}$ this is not always the case. EBV status has also been shown to not necessarily influence the genetic alterations seen in plasmablastic neoplasms. Yet, it is believed that EBV inhibits apoptosis and promotes pathogenesis in EBV-associated lymphoproliferative disorders. Further genetic and viral analysis will be required before PBM and PBL can be reliably distinguished.

PBL most often occurs in HIV-positive patients or those that are otherwise immunocompromised. PBM typically occurs in the elderly population, but has been rarely seen in aggressive forms in the young. ${ }^{11}$ Our patient did not have any overt causes of immunosuppression, but likely has immunosenescence due to his advanced age, which may have predisposed him to developing PBM.

Clinically, PBL is aggressive and has a poor prognosis, with an average survival time of 6 months from time of diagnosis. The survival of patients with PBM is similarly poor. In PBL, EBV positivity confers a better prognosis. Due to the overlapping features of PBM and PBL, our patient's EBV positive status may also prolong survival. C-MYC negativity in $\mathrm{PBL}$ also confers a better prognosis. The patient presented in this case report was also C-MYC negative, which in theory may also improve the patient's prognosis slightly. More research needs to be completed before such comparisons can be made, especially considering the poor prognosis of both disease entities. The diagnosis of either PBL or PBM is frequently difficult due to a diverse set of clinical presentations and similar morphological, immunochemistry profiles, and pathological features. ${ }^{12}$ Through further genetic and molecular analyses and viral studies, the cause of PBL and PBM may be elucidated and more clearly define treatment protocols.

\section{Acknowledgements}

None.

\section{Conflict of interest}

G. Monohan, M.D. reports equity interest outside of diversified mutual funds in Johnson \& Johnson, Novartis and Pfizer.

\section{References}

1. Vega F, Chang CC, Medeiros LJ, et al. Plasmablastic lymphomas and plasmablastic plasma cell myelomas have nearly identical immunophenotypic profiles. Mod Pathol. 2005;18(6):806-815.

2. Ishida $\mathrm{M}$, Hodohara $\mathrm{K}$, Okuno $\mathrm{H}$, et al. IgD plasmablastic myeloma: a case report with emphasis on the cytological features. Int J Clin Exp Pathol. 2014;7(3):1250-1254.

3. Reddy R, Vohra S, Macurak R. Secondary extramedullary plasmablastic myeloma of the small bowel. Clin Gastroenterol Hepatol. 2015;13(10):A23-A24

4. Bohn OL, Hsu K, Hyman DM, et al. BRAF V600E mutation and clonal evolution in a patient with relapsed refractory myeloma with plasmablastic differentiation. Clin Lymphoma Myeloma Leuk. 2014;14(2):e65-e68.

5. Kanungo A, Medeiros LJ, Abruzzo LV, et al. Lymphoid neoplasms associated with concurrent $\mathrm{t}(14 ; 18)$ and $8 \mathrm{q} 24 / \mathrm{c}-\mathrm{MYC}$ translocation generally have a poor prognosis. Mod Pathol. 2006;19(1):25-33.

6. Montes-Moreno S, Martinez-Magunacelaya N, Zecchini-Barrese T, et al. Plasmablastic lymphoma phenotype is determined by genetic alterations in MYC and PRDM1. Mod Pathol. 2017;30(1):85-94.

7. Igawa T, Sato Y, Kawai H, et al. Spontaneous regression of plasmablastic lymphoma in an elderly human immunodeficiency virus (HIV)-negative patient. Diagn Pathol. 2015;10:183.

8. Castillo JJ, Bibas M, Miranda RN. The biology and treatment of plasmablastic lymphoma. Blood. 2015;125(15):2323-2330. 
9. Hummel M, Anagnostopoulos I, Korbjuhn P, et al. Epstein-Barr virus in B-cell non-Hodgkin's lymphomas: unexpected infection patterns and different infection incidence in low- and high-grade types. J Pathol. 1995;175(3):263-271.

10. Carbone A, Gloghini A, Larocca LM, et al. Expression profile of MUM1/IRF4, BCL-6, and CD138/syndecan-1 defines novel histogenetic subsets of human immunodeficiency virus-related lymphomas. Blood. 2001;97(3):744-751.
11. Srija M, Zachariah PP, Unni VN, et al. Plasmablastic myeloma presenting as rapidly progressive renal failure in a young adult. Indian J Nephrol. 2014;24(1):41-44.

12. Elyamany G, Alzahrani AM, Aljuboury M, et al. Clinicopathologic features of plasmablastic lymphoma: Single-center series of 8 cases from Saudi Arabia. Diagn Pathol. 2015;10:78. 